\title{
Photoluminescence of Diamondoid Crystals
}

\author{
William A. Clay ${ }^{1}$, Takao Sasagawa ${ }^{2}$, Akio Iwasa ${ }^{2}$, Zhi Liu ${ }^{3}$, Jeremy E. Dahl ${ }^{1}$, \\ Robert M. K. Carlson ${ }^{4}$, Michael Kelly ${ }^{1}$, Nicholas Melosh ${ }^{1}$, and Zhi-Xun Shen ${ }^{1,5}$ \\ ${ }^{1}$ Department of Physics, Applied Physics, and Geballe Laboratory for \\ Advanced Materials, Stanford University, Stanford, CA 94305 USA \\ ${ }^{2}$ Materials and Structures Laboratory, Tokyo Institute of Technology, Midori-Ku, Yokohama, 226-8503 Japan \\ ${ }^{3}$ Advanced Light Source, Lawrence Berkeley Laboratory, Berkeley, CA 94550, USA \\ ${ }^{4}$ MolecularDiamond Technologies, Chevron Technology Ventures, \\ 100 Chevron Way, Richmond, CA 94802, USA and \\ ${ }^{5}$ Stanford Institute for Materials and Energy Sciences, \\ SLAC National Accelerator Laboratory, 2575 Sand Hill Road, Menlo Park, CA 94025, USA
}

(Dated: September 21, 2011)

The photoluminescence of diamondoids in the solid state is examined. All of the diamondoids are found to photoluminesce readily with initial excitation wavelengths ranging from $233 \mathrm{~nm}$ to $240 \mathrm{~nm}(5.3 \mathrm{eV})$. These excitation energies are more than $1 \mathrm{eV}$ lower than any previously studied saturated hydrocarbon material. The emission is found to be heavily shifted from the absorption, with emission wavelengths of roughly $295 \mathrm{~nm}(4.2 \mathrm{eV})$ in all cases. In the dissolved state, however, no fluorescence is observed for excitation wavelengths as short as $200 \mathrm{~nm}$. We also discuss predictions and measurements of the quantum yield. Our predictions indicate that the maximum yield may be as high as $25 \%$. Our measurement of one species, diamantane, gives a yield of $11 \%$, the highest ever reported for a saturated hydrocarbon, even though it was likely not at the optimal excitation wavelength.

\section{INTRODUCTION}

Diamond-like hydrocarbon molecules, also known as diamondoids, are a unique system of carbon nanoparticles. Interest in these unusual molecules has increased greatly since the discovery of previously unobtainable quantities of these molecules ${ }^{1}$. In addition to the fact that diamondoids inherit many of the desirable properties of bulk diamond ${ }^{2-5}$, they display a number of interesting physical attributes which come from their shape, high surface ratio, and/or nanometer-size $\mathrm{e}^{5-14}$, as well as unique chemical properties ${ }^{15-17}$. We present a study of their photoluminescence, which exhibits a number of interesting properties. 
Diamondoids readily form macroscopic molecular crystals at atmospheric temperatures and pressures, and it is the photoluminescence of these molecular crystals which we study here. We find that the crystals exhibit photoluminescence which is considerably different than that observed from the isolated molecules when measured in solution ${ }^{18}$ or in the gas phase ${ }^{19}$. All of the crystalline samples exhibit qualitatively similar emission spectra, with emission peaks of roughly $295 \mathrm{~nm}$. All samples also show similar absorption thresholds, with threshold wavelengths between 233 and $240 \mathrm{~nm}$ in all cases. The apparent lack of quantum confinement related to the size of the molecules is an interesting property of the bulk state of these molecular crystals.

Although photoluminescence has been previously observed in saturated hydrocarbons in the liquid state ${ }^{20-22}$, this is the first study of a saturated hydrocarbon showing a condensed-state photoluminescence which is considerably different from the gas or solution phase behavior of the same compound. Additionally, we find that the minimum excitation energy required for photoluminescence is significantly lower than that of other saturated hydrocarbons. We also predict that these crystals should have significantly higher yield than other saturated hydrocarbons, and we have measured a significantly higher yield than has been observed in such systems before. The reason for these unusual properties is the unusual structure of the diamondoids. Unlike nearly all other classes of saturated hydrocarbons, diamondoids have a rigid structure which allows no internal rotation or conformational changes, and yet have very little strain ${ }^{23}$. This gives them unique electrical and chemical properties which are not present in the other similar system whose fluorescence has been studied.

\section{EXPERIMENTAL METHODS}

Samples of isolated diamondoids were obtained in a powdered crystalline form from MolecularDiamond Technology. The samples were had been pre-treated by the supplier through pyrolysis and high pressure liquid chromatography (HPLC) to isolate and separate the diamondoids, and the samples were of greater than 99\% purity. These samples were further purified using either argentic HPLC or by repeated sublimation to remove any aromatic impurities from the sample ${ }^{18}$. This step was critical because the diamondoid samples, which are derived from crude oil ${ }^{1}$, contained trace impurities of various naphthalene derivatives which fluoresce strongly under UV light. Argentic HPLC was used because the silver helped to strongly filter out any molecules containing double bonds. Triamantane and tetramantane were purified using the HPLC method for one run and the repeated crystallization method for a second run, with no apparent difference in results. Adamantane and diamantane were purified using the recrystallization method only.

Using these purified materials, fluorescence samples were made by pressing the powders or crystals into a flat puck 
in a mechanical press at a pressure of $15 \mathrm{MPa}$ or by using the crystals directly if they were of sufficient size. After measurement, these samples were also dissolved in fluorescence grade n-hexane to attempt to measure fluorescence in the solution state. For comparison, unpurified, as-received samples were also measured in the same way. All solutions were approximately $1 \mathrm{mmol} / \mathrm{L}$ concentration.

Photoluminescence spectra were recorded using a Hitachi F7000 fluorescence spectrophotometer equipped with a Xe lamp. This system has variable excitation wavelengths ranging from $200 \mathrm{~nm}$ to $500 \mathrm{~nm}$. Additional data on [121]tetramantane were taken using a OceanOptics USB2000 spectrophotometer and using a Coherent Innova 300 FreD argon gas laser equipped with a frequency doubling crystal as a light source. This second system, which had a minimum wavelength of $229.0 \mathrm{~nm}$, was used to verify the results. For the laser system, the samples were not crushed into pucks but were measured as bulk crystals. The data presented here are all taken on samples purified using the repeated recrystallization method, with the exception of the [121]tetramantane laser data, which was purified using repeated argentic HPLC.

The species measured were adamantane $\left(\mathrm{C}_{10} \mathrm{H}_{16}\right)$, diamantane $\left(\mathrm{C}_{14} \mathrm{H}_{20}\right)$, triamantane $\left(\mathrm{C}_{18} \mathrm{H}_{24}\right)$, and [121] tetramantane $\left(\mathrm{C}_{22} \mathrm{H}_{28}\right)$. We note that the diamondoids are named based on the number of diamond cages present in each species, with adamantane containing 1, diamantane containing 2, and so on. For structures with more than 3 cages the Balaban-Schleyer nomenclature is used to differentiate between isomers ${ }^{24}$.

Additionally, several attempts were made to measure the quantum yield. These measurements were performed using a JASCO FP-6500 spectrofluorimeter equipped with a Xenon lamp and a $\phi 100 \mathrm{~mm}$ Spectralon coated integrating sphere (JASCO ILF-533). We attempted to measure each species multiple times, but were only able to get a good enough signal-to-noise ratio on one of the measurements of diamantane. The difficulty with this measurement is that both the lamp intensity and the reflectivity of the integrating sphere coating become progressively worse at wavelengths below $250 \mathrm{~nm}$, combined with the fact that the diamondoid crystals generally scatter more light than they absorb near the absorption threshold. This resulted in extremely noisy data and poor statistics in most measurements. It was challenging to create samples which absorbed enough of the light to make the diamonoid emission peak stand out above the large background, and we are unsure why one diamantane sample provided better results than the other samples, which included other diamantane samples. 


\section{RESULTS AND DISCUSSION}

Using the Hitachi F7000 system, it was possible to create 3-D maps of the intensity as a function of both the excitation and emission wavelength. The map for solid state [121]tetramantane is shown as an example in figure 1a. No fluorescence was observed in the solution phase for any of the samples, as reported previously ${ }^{18}$. This is significant because solution phase fluorescence is the most sensitive technique available for detecting fluorescent impurities. Although the concentration of these impurities would be lower in solution than in the solid state, the solution measurement was much more sensitive for the following reasons: first, there was vastly lower background as there was no fluorescence from the sample holder and, more importantly, very little background from scattered incident light, which was the main source of noise in the solid samples. Second, the optical path in the solution cell was $1 \mathrm{~cm}$, which is more than 10 times the path through any of the solid samples. Therefore it would be very surprising if we were able to detect an impurity peak in the solid state measurement but not in the solution measurement. For example, When we measure the impure samples, we find that the main impurity peak (around $340 \mathrm{~nm}$ ) is of roughly the same intensity as the sample peak at $300 \mathrm{~nm}$ in the solid state, but in solution we can only detect the $340 \mathrm{~nm}$ impurity peak. In fact, the signal to noise ratio is much better for the $340 \mathrm{~nm}$ peak in solution, but the $300 \mathrm{~nm}$ peak is no longer detectable. This disparity would not make sense if both peaks were derived from trace impurities. This behavior makes sense, however, if we assume that the $300 \mathrm{~nm}$ peak comes from the diamondoid. We are unable to reach the optical band gap of the diamondoids in a diffuse state ${ }^{25}$ with our apparatus, so it is not surprising that we see no the fluorescence in the dissolved state.

Figure 1b shows cross sections of this data taken at 220 and $244 \mathrm{~nm}$ excitation. Figure 1c shows a cross section of this data as well, with the excitation wavelength $\lambda_{e x}$ at $228 \mathrm{~nm}$, compared with the laser system spectrum at 229.0 nm. Figure 1d shows the intensity as a function of excitation energy for a single emission wavelength, $300 \mathrm{~nm}$. In the solid state color map (figure 1a) we can see the threshold wavelength $\lambda_{t h}$ below which fluorescence is observed at $239 \mathrm{~nm}$. We see that below this wavelength, the intensity of the spectrum increases with decreasing $\lambda_{e x}$, but the peak position and the width are not detectably changed as we vary the excitation. In the cross section in figure $1 \mathrm{~b}$ we can see the shape of the spectrum, both above and below threshold. The low-intensity peak in the below-threshold spectrum (244 nm excitation) is almost certainly background from the sample holder, which is impossible to subtract perfectly. In the cross section at $228 \mathrm{~nm}$ excitation (figure 1c), we see that the Xe lamp data are in close agreement with the laser data. The slight discrepancy is most likely caused by either noise or small sample holder background in the fluorescence spectrometer data, which may distort the peak shape slightly. 
Similar data were taken on the fluorescence spectrometer for the other diamondoids, and the results were qualitatively and quantitatively quite similar in all cases. The emission curve for $\lambda_{e x}=220 \mathrm{~nm}$ and the excitation curve for $\lambda_{e m}=300 \mathrm{~nm}$ are shown for each species measured in figure 2. As is seen, all of the peaks are quite similar with peak maximums $\lambda_{\max }$ in the range from 293-299 $\mathrm{nm}$ and full width at half maximum (FWHM) values of 35 to 50 $\mathrm{nm}$. The emission threshold was also very similar in all cases, with $\lambda_{\text {threshold }}$ values ranging from $233-240 \mathrm{~nm}$.

The results of the quantum yield measurement of diamantane are shown in figure 3 . As is clear, the absorbance in the diamondoid is not very high and the spectrum is very noisy. Based on this spectrum, we estimate that the quantum yield of diamantane (at $220 \mathrm{~nm}$ excitation) to be $11 \pm 4 \%$. Despite the large uncertainty, this is exceptionally high for a saturated hydrocarbon. The highest previously measured was $2.2 \%^{20,22}$. Additionally, based on the excitation energy curves seen in figure 2, this is probably not the excitation energy which yields the maximum quantum yield. This was the lowest wavelength that could be worked with using our apparatus, however, so we were unable to probe shorter excitation wavelengths.

In many respects, the fluorescence of the diamondoids is quite similar to that of other saturated hydrocarbons, as described in detail in $\mathrm{ref}^{22}$. FWHMs of roughly $40 \mathrm{~nm}$ and excitation-emission shifts in the range of .58 to $1.29 \mathrm{eV}$ are typical in other saturated hydrocarbons. Both of these properties are believed to be caused by nuclear distortions of the molecule's atoms in the excited state ${ }^{22}$, and we assume that this is the case for diamondoids as well. The slight asymmetry in the spectra is evidence of coupling to phonons in the crystal, which may contribute somewhat to the spectral broadening as well. This asymmetry is not observed in other saturated hydrocarbons, but this is to be expected because they were measured in the liquid state.

Additionally, it is quite common for the emission wavelength $\lambda_{\max }$ to be fairly constant within a class of saturated hydrocarbon materials. Among unbranched alkanes, for example, the value of $\lambda_{\text {max }}$ varies only from 206 to $208 \mathrm{~nm}$ as the size of the chain is varied from 5 carbons to $16^{22}$. The general trend of higher excitation and emission energy for smaller molecules is consistent with the expectation that quantum confinement will increase the energy of the excited states on these smaller molecules. Given the nanometer scale size of the individual molecules, however, one might expect that quantum confinement would play a stronger role in determining the absorption and emission properties. Indeed, single molecule band gap computations indicate that the isolated molecules should exhibit stronger quantum confinement effects $^{26}$. According to solid state (bulk crystal) computations, however, diamondoids show a remarkable amount of wavefunction overlap, resulting in the formation of diffuse bulk crystal bands ${ }^{11}$, which greatly lessens the role of quantum confinement in the excited states of the crystalline state. 
Diamondoid photoluminescence is similar in many ways to that of other saturated hydrocarbons, and indeed the gas phase data for adamantane ${ }^{19}$ is virtually indistinguishable from other similar molecules. In the solid state, however, diamondoid crystals exhibit a number of photoluminescence properties which set them apart from previously studied saturated hydrocarbon materials. Their excitation and emission energy are both more than $1.0 \mathrm{eV}$ lower than the lowest previously reported species $^{22}$. They also have a significant difference between the diffuse (gas or solution) behavior and the condensed state behavior. Such behaviors are far more common in conjugated aromatic materials, and not previously observed in a system with no double bonds whatsoever. In most organic crystal fluors, the doublebond orbitals (also known as the pi system) are critical to the fluorescence process ${ }^{27}$. The presence of these heavily hybridized orbitals results in a large reduction in the HOMO-LUMO gap relative to saturated systems and also allows for a large degree of charge delocalization. These factors result in relatively low energy, high stability excited states which are conducive to photoluminescence.

In most saturated systems, on the other hand, the HOMO-LUMO gap is quite large, resulting in minimum excitation energies greater than $6.5 \mathrm{eV}$ in nearly all cases ${ }^{20-22}$. Additionally, the orbitals are well localized to individual bonds. This means that the excited state is usually high energy and very unstable. Typically it induces strong deformations in the shape of the molecule ${ }^{22,28}$. The end result is that the photoluminescence process is weak, with yields less (typically much less) than $2.2 \%^{20,22}$. The most common channel for de-excitation in other saturated hydrocarbons is instead photofragmentation via a reverse hydrogenation reaction, with a hydrogen molecule breaking away from the parent molecule and leaving a double bond behind ${ }^{28}$. Additionally, the high degree of charge localization means that there is little advantage to the solid state over the gas or solution phase, because there is little hybridization of orbitals and therefore little reduction in band-gap.

To understand what makes diamondoids unique, we must look at what makes them different than all typical saturated hydrocarbons. The answer is the rigid, diamond-like structure which defines this class of molecules. Because diamondoid molecules have no internal degrees of rotation, the position of the atomic cores is essentially fixed within the molecule. This allows the formation of diffuse molecular orbitals, even without the existence of a pi system, and makes it possible to easily form ordered crystals. These diffuse orbitals have been predicted in single molecule ${ }^{8}$ and bulk $\mathrm{DFT}^{11}$ calculations and have been observed directly in SEM spectroscopy ${ }^{29}$. Although the optical gap is significantly larger than that of aromatic systems, it is still noticeably smaller than other saturated hydrocarbons. It has been measured to be between $5.5 \mathrm{eV}$ and $6.0 \mathrm{eV}^{7}$ in the solid state using x-ray absorbance and emission. In these measurements, the optical gap is found to decrease with increased molecular size, with values of $6.0 \mathrm{eV}$ 
for adamantane, $5.8 \mathrm{eV}$ for diamantane, $5.7 \mathrm{eV}$ for triamantane, and $5.6 \mathrm{eV}$ for tetramantane. In addition to this decrease in the gap, these fixed atomic cores and delocalized orbitals also result in other properties which resemble the aromatics more than other saturated systems. First, these properties allow diamondoids to form good, ordered crystals at room temperature, which is not observed in any other saturated hydrocarbon whose fluorescence has been studied, all of which are liquids at room temperature. Second, it allows for further wave function hybridization as the diffuse molecular orbitals further hybridize into crystal wavefunctions. This results in a large reduction in the band gap in the condensed phase, typically about $1 \mathrm{eV}^{11}$ according to DFT studies. This fact is evident from the large shift between the fluorescence onset in the crystals as measured here and the gas phase absorption curves ${ }^{25}$, and explains why the low excitation threshold is only present in the solid state. The evident lack of quantum confinement effects can be explained using these predicted band structures if we assume that the "hole" part of the excitation, which inhabits the normally occupied states, is well confined in all cases (as is predicted from the band structure) while the "electron" part, which inhabits the normally unoccupied states, is extremely diffuse in all cases. This model only makes sense, however, if we assume that the hole is always extremely well localized, perhaps to a single bond or atom, such that the molecule size does not greatly effect its energy. This is consistent with the simulations, however, which always shows a well-localized HOMO even in the ground state.

Given their properties, we expect that the diamondoids should have an unusually high quantum yield compared with other saturated systems. From existing data on saturated hydrocarbons ${ }^{22,30}$, we have observed a strong correlation between the excitation threshold $E_{t h}$ and the maximum value of the quantum yield $\phi_{\max }$. The relation is plotted in figure 4 for n-decane $\left(\mathrm{C}_{10} \mathrm{H}_{22}\right)$, n-dodecane $\left(\mathrm{C}_{12} \mathrm{H}_{26}\right)$, n-pentadecane $\left(\mathrm{C}_{15} \mathrm{H}_{32}\right)$, 2,3-dimethylbutane $\left(\mathrm{C}_{6} \mathrm{H}_{14}\right)$, cyclohexane $\left(\mathrm{C}_{6} \mathrm{H}_{12}\right)$, methylcyclohexane $\left(\mathrm{C}_{7} \mathrm{H}_{14}\right)$, bicyclohexyl $\left(\mathrm{C}_{12} \mathrm{H}_{20}\right)$, cis-decalin $\left(\mathrm{C}_{10} \mathrm{H}_{18}\right)$, and trans-decalin $\left(\mathrm{C}_{10} \mathrm{H}_{18}\right)$, which were all of the reasonably fluorescent samples available in both sources. The reason for this trend is presumably that the less energy the system has in the lowest excited singlet state, the less likely it is that the molecule will undergo photofragmentation. $\mathrm{H}_{2}$ Photofragmentation is an endothermic process which has a high activation energy. We know this because the reverse reaction, the hydrogenation reaction, is highly exothermic but does not occur at an observable rate even at elevated temperatures without the presence of a catalyst. Because of the high activation energy, it is a reasonable assumption that the hydrogen atoms pass though a classically forbidden region, which means that the process can be approximated as a tunneling process. We can approximate the tunneling probability if we assume that the tunnel barrier has a height $E_{A}$ above the ground state and approximate its shape as a parabola. Using the standard WKB tunneling approximation with this potential barrier, we can then calculate the tunneling probability 


$$
P_{\text {photolysis }} \approx \exp \left(C_{0} \int_{-\sqrt{\frac{\sigma}{E^{*}}}}^{\sqrt{\frac{\sigma}{E^{*}}}} \sqrt{E^{*}-\sigma x^{2}} d x\right)
$$

where $C_{0}$ is a fundamental constant related to the mass, $E^{*}$ is $E_{A}-E_{t h}$, and $\sigma$ defines the tunnel barrier width. If we then make the further assumption that the fluorescence rate is equal for all species (which is not too far from accurate $^{31}$ ), then the photofragmentation yield should be equal to this tunneling probability, which is proportional to the tunneling rate. Because the total yield is 1 , our photoluminescence yield, $\phi$ is equal to $1-P_{\text {photolysis }}$, and we can solve the integral and rearrange the previous equation to yield the following relation between the quantum yield $(\phi)$ and the threshold energy.

$$
\ln (1-\phi)=-C_{1}\left(E_{A}-E_{t h}\right)
$$

Where $C_{1}$ is a constant related to the unknown width of the tunnel barrier. We plot this relation in the inset of figure 4 along with a linear fit. This analysis predicts a quantum yield of 6-6.3 for the quantum yield of a species with $\lambda_{t h}$ of 233-239, the values for the diamondoids.

As side note, this analysis predicts an approximate activation energy for reverse hydrogenation of $7.4 \mathrm{eV}$. This prediction would imply that fluorescence yields should be significantly suppressed for species with $E_{A}$ close to $7.4 \mathrm{eV}$, and indeed the highest reported threshold energy of a fluorescent species in the literature is $7.3 \mathrm{eV}^{22}$.

Additionally, the rigid structure of diamondoids means that $\mathrm{H}_{2}$ photofragmentation will be energetically unfavorable because there is no way to form a stable double bond on the surface of a diamondoid molecule; the bond will be too strained because the pi orbitals will not overlap well at all unless one of the carbon-carbon bonds breaks as well. This means that the only non-radiative de-excitation method possible for the diamondoids is the less favorable single hydrogen atom photofragmentation. Because of this, we predict that diamondoids should have significantly higher quantum yields than other saturated hydrocarbons. Given that the typical ratios of yields for $\mathrm{H}_{2}$ fragmentation: $\mathrm{H}$ fragmentation:photoluminescence is about 83:16:128 near the absorption threshold, we predict that the yield for the diamondoids could be more than 5 times higher than other similar species. Combining this with our other prediction of the yield using the threshold energy, we predict that yields as high as 25-30\% may be possible in some diamondoids.

We do note that the measured quantum yield value for diamantane $(11 \%)$ is higher than the extrapolated value for a standard hydrocarbon, but is significantly lower than the upper limit of our predictions. There are several reasons why this may be the case. First, the excitation energy used to measure the quantum yield was probably not ideal. The maximum value of the yield (which is what is used to fit the model above) may be somewhat higher. There 
is typically 1.5 to $1.8 \mathrm{eV}$ difference between the fluorescence onset energy and the energy that gives the quantum yield maximum in saturated hydrocarbons ${ }^{30}$, which would imply that the maximum quantum yield would occur at an excitation of about $180 \mathrm{~nm}$, which is outside the range of our measurement capacity. There may be additional factors which reduce the yield, however, and we cannot rule any of them out. For example, there may be non-radiative decay modes which are unique to the solid state, such as de-excitation through coupling to bulk phonons. It is also possible that the diamondoids have slightly reduced quantum yields due to bond strain, which generally reduces fluorescence yields $^{22}$. Although the diamondoids have very little strain compared to other cage hydrocarbons, they are not strain free $e^{23,32}$, and this strain may reduce the quantum yield slightly.

\section{CONCLUSIONS}

Using several techniques, we were able to observe the fluorescence spectra of the diamondoids in their solid, crystalline state. In many ways the fluorescence of these unusual molecules is quite similar to that of other saturated hydrocarbons. The value of the peak width and the shift between the absorbed and emitted wavelength are both quite similar to other such species. To our knowledge, however, the diamondoids exhibit the lowest energy for both excitation and emission for any saturated hydrocarbon by a significant amount. Additionally, to our knowledge, this is the first discovery of a fluorescence in a saturated hydrocarbon which is essentially unique to the solid state and not observed in a diffuse state. According to our predictions, the diamondoids should also exhibit by far the highest quantum yield of any saturated hydrocarbon, and our measurements support this conclusion.

\section{Acknowledgments}

This work was supported, in part, by a grant from Chevron through the Stanford-Chevron Diamondoid program, as well as by the DOE Office of Basic Energy Science, Division of Material Science and Engineering under contract DE-AC02-76SF00515. Work performed at the Tokyo Institute of Technology was supported by a research grant from Iketani Science and Technology Foundation and a Grant-in-Aid for Scientific Research from MEXT, Japan. 
1 J.E. Dahl, S.G. Liu and R. M. K. Carlson, Science 29996 (2003)

2 P. Badziag, W. S. Verwoerd, W. P. Ellis and N. R. Greiner, Nature 343244 (1990)

${ }^{3}$ M. Linnolahti, A. J. Kartunnen, T. A. Pakkanen, J. Phys. Chem. C 11118118 (2007)

${ }^{4}$ R. C. Fort Adamantane: The Chemistry of Diamond Molecules (Marcel Dekker Inc. New York, NY, 1976)

${ }^{5}$ H. Schwertfeger, A. A. Fokin and P. R. Schreiner, Angew. Chem. Int. Ed. 471022 (2008)

${ }^{6}$ T. M. Willey, C. Bostedt, T. van Buuren, J. E. Dahl, S. G. Liu, R. M. K. Carlson, L. J. Terminello and T. Moller, Phys. Rev. Lett. 95113401 (2005)

7 T. M. Willey, C. Bostedt, T. van Buuren, J. E. Dahl, S. G. Liu, R. M. K. Carlson, R. W. Meulenberg, E. J. Nelson and L. J. Terminello, Phys. Rev. B 74205432 (2006)

8 G. C McIntosh, M. Yoon, S. Berber and D. Tomanek, Phys. Rev. B 70045401 (2004)

${ }^{9}$ N. D. Drummond, A. J. Williamson, R. J. Needs and H. Galli, Phys. Rev. Lett. 95096801 (2005)

10 W. L. Yang, J. D. Fabbri, T. M. Willey, J. R. I. Lee, J. E. Dahl, R. M. K. Carlson, P. R. Schreiner, A. A. Fokin, B. A. Tkachenko, N, A, Fokina, W. Meevasana, N. Mannella, K. Tanaka, X. J. Zhou, T. van Buuren, M. A. Kelly, Z. Hussain, N. A. Melosh and Z.-X. Shen, Science 3161460 (2007)

11 T. Sasagawa and Z.-X. Shen, J. Appl. Phys. 104073704 (2008)

${ }^{12}$ W. A Clay, T. Sasagawa, M. Kelly, J. E. Dahl, R. M. K. Carlson, N. Melosh and Z.-X. Shen, Appl. Phys. Lett. 93 $172901(2008)$

${ }^{13}$ W. A. Clay, Z. Liu, WL. Yang, J. D. Fabbri, J. E. Dahl, R. M. K. Carlson, Y. Sun, P. R. Schreiner, A. A. Fokin, B. A. Tkachenko, N. A. Fokina, P. A. Pianetta, N. Melosh and Z.-X. Shen, Nanolett. 957 (2009)

${ }^{14}$ W. Meevasana, R. Supruangnet, H. Nakajima, O. Topon, V. Amornkitbamrung and P. Songsiriritthigul, Appl. Surf. Sci. 256934 (2009)

15 A. A. Fokin, B. A. Tkachenko, B. A. Gunchenko, D. V. Gusev and P. R. Schreiner, Chem.-Eur. J. 117091 (2005)

16 P. R. Schreiner, N. A. Fokina, B. A. Tkachenko, H. Hausmann, M. Serafin, J. E. Dalh, S.G. Liu, R. M. K. Carlson and A. A. Fokin, J. Org. Chem. 716709 (2006)

17 B. A. Tkachenko, N. A. Fokina, L. V. Chernish, J. E. P. Dahl, S. G. Liu, R. M. K. Carlson, A. A. Fokin and P. R. Schreiner, Org. Lett. 81767 (2006)

18 A. Iwasa, W. A. Clay, J. E. Dahl, R. M. K, Carlson, Z.-X. Shen and T. Sasagawa, Cryst. Growth Des. 10870 (2010)

${ }^{19}$ L. Landt, W. Kielich, D. Wolter, M. Staiger, A. Ehresmann, T. Moller and C. Bostedt, Phys. Rev. B 80 205323 (2009)

${ }^{20}$ F. Hirayama and S. Lipski, J. Chem. Phys. 513616 (1969)

${ }^{21}$ F. Hirayama, W. Rothman and S. Lipski, Chem. Phys. Lett. 5296 (1970) 
${ }^{22}$ W. Rothman, F. Hirayama and S. Lipski, J. Chem. Phys. 581300 (1973)

${ }^{23}$ P. von R. Schleyer, J. E. Williams and K. R. Blanchard, J. Am. Chem. Soc. 922377 (1970)

24 A. T. Balaban and P. von R. Schleyer, Tetrahedron 343599 (1978)

${ }^{25}$ L. Landt, K. Klunder, J. E. Dahl, R. M. K. Carlson, T. Moller and C. Bostedt, Phys. Rev. Lett. 103 047402 (2009)

${ }^{26}$ A. A. Fokin and P. R. Schreiner, Mol. Phys. 107823 (2009)

27 R. M. Hochstrasser, Rev. Mod. Phys. 34531 (1962)

${ }^{28}$ F. P. Schwarz, D. Smith, S. G. Lias and P. Ausloos, J. Chem. Phys. 753800 (1981)

${ }^{29}$ Y. Wang, E. Kiopakis, X. Lu, D. Wegner, R. Yamachika, J. E. Dahl, R. M. K. Carlson, S. G. Louie and M. F. Crommie, Nature Mat. 738 (2008)

30 A. E. Ostafin and S. Lipski, J. Chem. Phys. 985408 (1993)

31 W. R. Ware and R. L. Lyke, Chem. Phys. Lett. 24195 (1974)

32 T. Clark, T. M. Knox, M. A. McKervey, H. Mackle and J. J. Rooney, J. Am. Chem. Soc. 1012404 (1979) 

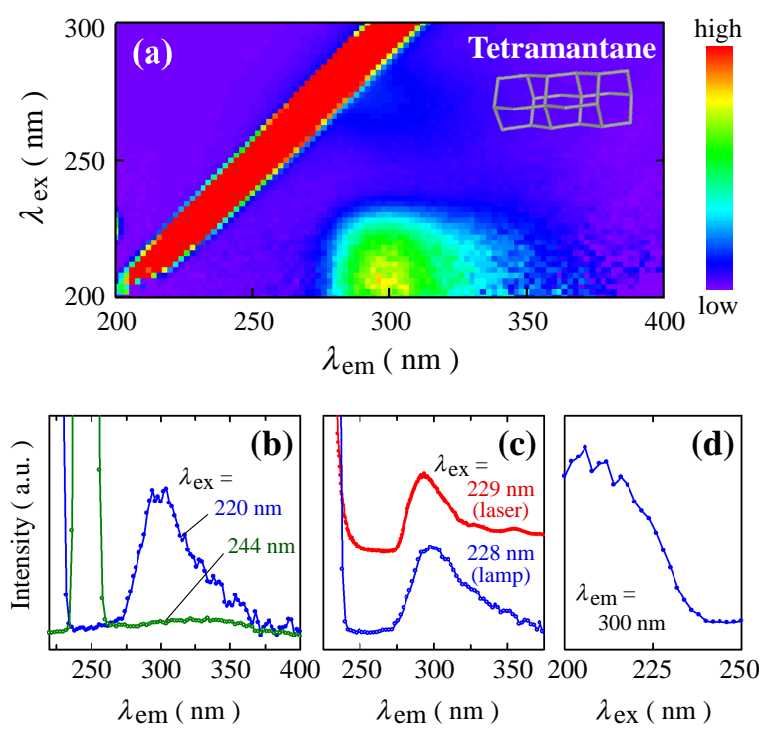

FIG. 1: a) the intensity map for fluorescence from [121]Tetramantane as a function of both emission and excitation wavelength.

b) Cross section spectra for single excitation energies. Cross sections are shown for 220 and 244 nm excitations. c) Comparison of data taken using Laser system at $229.0 \mathrm{~nm}$ with data from the fluorescence spectrophotometer at $228 \mathrm{~nm}$. d) Cross section spectra for a single emission wavelength, at $300 \mathrm{~nm}$ 


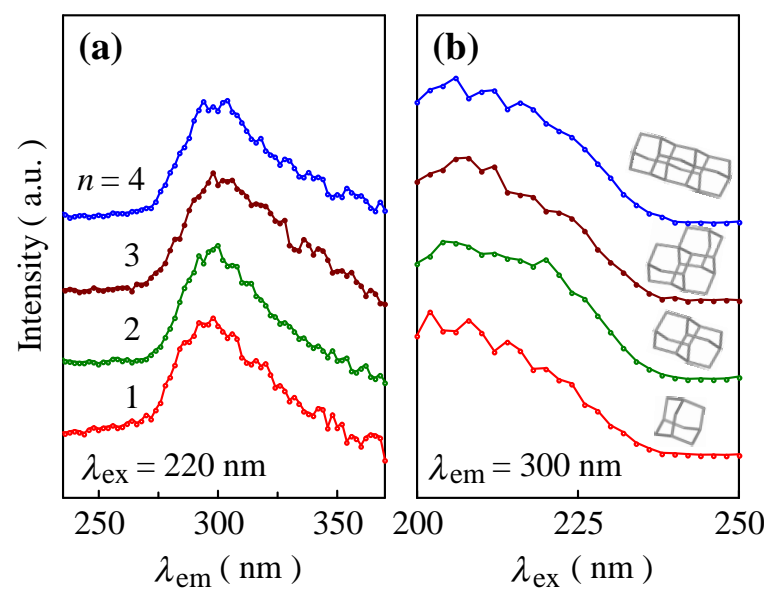

FIG. 2: a) Emission peaks and b) excitation peaks from adamantane, diamantane, triamantane, and [121]tetramantane. $n$ refers to the number of diamond cages in the diamondoid molecule. Spectra are normalized to peak height and offset for clarity. 


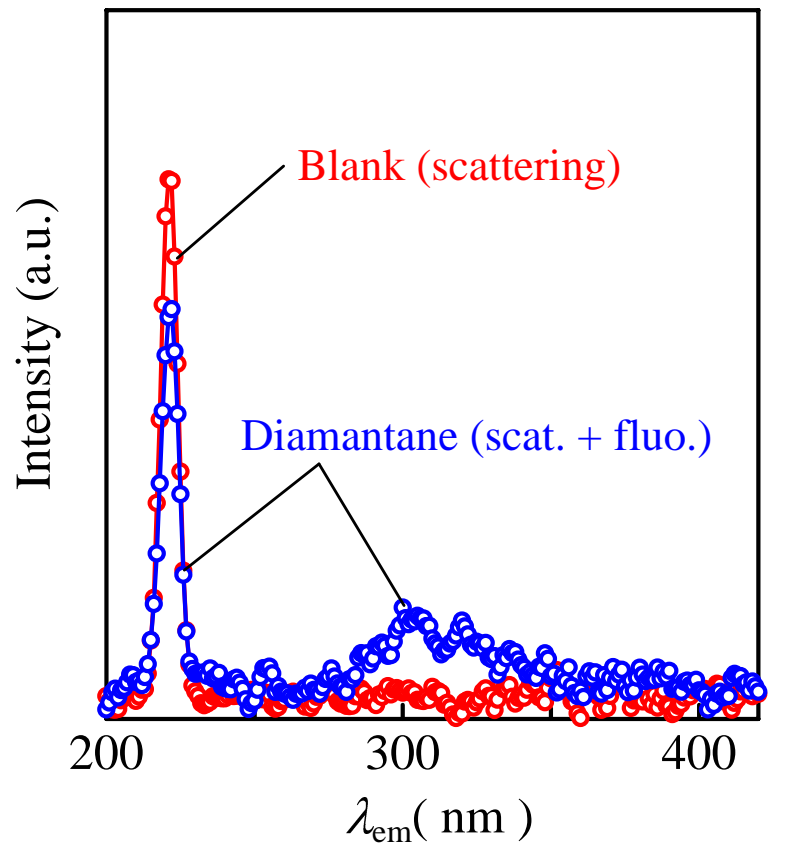

FIG. 3: Quantum yield measurement for diamantane crystal. Red curve shows control with just scattering off the sample holder, blue curve shows absorption and emission from diamantane. Measured quantum yield is $11 \pm 4 \%$ 


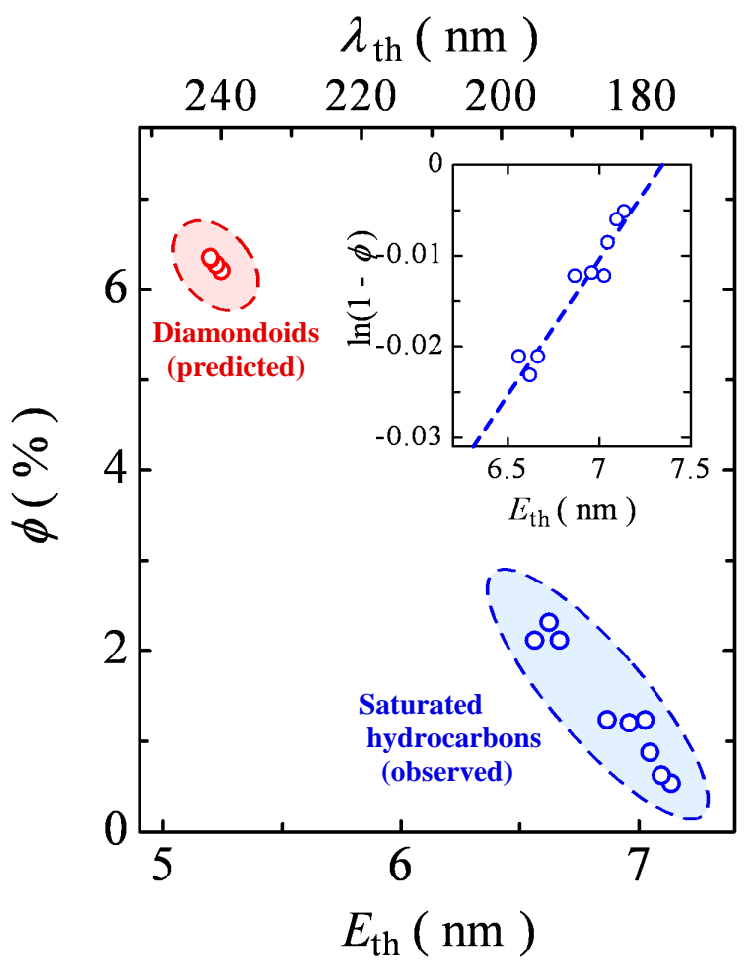

FIG. 4: Quantum yield as a function energy threshold for 9 saturated hydrocarbon species, using data from refs ${ }^{22,30}$, along with predicted values for diamondoids. Inset shows data fitted to theoretical model. From lowest threshold to highest, these are cis-decalin, trans-decalin, bicyclohexyl, methyl-cyclohexane, cyclohexane, 2,3-dimethylbutane, n-pentadecane, n-dodecane, and n-decane 